\title{
Análisis geográfico del matrimonio entre personas del mismo sexo en España: evolución, distribución y perfiles tras una década de legalidad
}

Geographical analysis of same-sex marriage in Spain: evolution, distribution and profiles after a decade of legality

\author{
Alberto Capote Lama \\ alama@ugr.es \\ Departamento de Geografía Humana \\ Universidad de Granada (España) \\ José Antonio Nieto Calmaestra \\ jantonio.nieto@juntadeandalucia.es \\ Delegación Territorial de Medio Ambiente \\ y Ordenación del Territorio en Granada (España)
}

\section{Resumen}

En 2005 España pasó a ser el tercer país de la Unión Europea en legalizar el matrimonio entre personas del mismo sexo. Una vez superado el simbólico umbral del décimo aniversario, parece un momento oportuno para hacer un balance y ver cuál ha sido la evolución de este tipo de evento nupcial desde su aparición, así como su distribución geográfica y los perfiles más comunes entre sus protagonistas. El análisis, a partir de las principales fuentes estadísticas disponibles, pone de relieve que se trata de una realidad bastante asentada en la sociedad española, aunque con un 
notable nivel de concentración geográfica. La necesidad de integrar una perspectiva de género, cuando se estudia el matrimonio entre personas del mismo sexo, se hace también evidente.

Palabras clave: matrimonio homosexual; España; distribución geográfica; evolución; perfil sociodemográfico.

\begin{abstract}
In 2005 Spain became the third EU country to legalize same-sex marriage. Once the symbolic 10year threshold anniversary has passed, it is an appropriate moment to make balance and see the evolution of this kind of nuptial event since its appearance, its geographical distribution as well as the most common profile of its protagonists. The analysis carried out from the main statistical sources shows how it has become a fairly established reality in Spanish society, although with a remarkable level of geographical concentration. The need to integrate a gender perspective when studying same-sex marriage is also evident.
\end{abstract}

Key words: gay marriage; Spain; geographical distribution; evolution; sociodemographic profile.

\title{
1 Introducción
}

En el año 2005 España pasó a ser el tercer país de la Unión Europea, después de Holanda y de Bélgica, que legalizó el matrimonio entre personas del mismo sexo (Ley 13/2005, de 1 de julio), una de las reformas jurídico-sociales más trascendentales de los últimos años (Soriano Martínez, 2011). La legislación del matrimonio entre personas del mismo sexo tuvo lugar en un contexto en el que los cambios en la familia española se estaban consolidando, situaciones que eran compartidas con otras sociedades del entorno, aunque en España se empezaron a desarrollar más tardíamente: caída de la nupcialidad, aumento de la fragilidad en las uniones, aparición de una mayor pluralidad de fórmulas de convivencia, proceso creciente de secularización de la familia, etc. (Castro Martín \& Seiz Puyuelo, 2014; Domínguez Folgueras, 2011; Requena, 2008, 2009). Castro Martín (2015) sitúa estas transformaciones familiares y cambios demográficos en la denominada segunda transición demográfica que se observa en algunas sociedades europeas desde los años sesenta, si bien en el caso español se produce algo más tarde, aunque de manera más intensa. En el contexto de dicha transición, Jurado (2008) destaca que España, a partir de los noventa, se muestra muy tolerante hacia las relaciones homosexuales, incluso más que en otros países vecinos. En esta línea, para Gimeno y Barrientos (2009), la legislación de las uniones entre personas del mismo sexo contribuyó de forma sustancial a estos cambios ya que hizo más visible la ruptura de una concepción naturalista del matrimonio: es la propia sociedad en cada momento la que determina lo que quiere que sea una unión matrimonial, sin preconcepciones religiosas de ningún tipo. Grau Rebollo (2016) también pone de relieve que la visibilidad creciente de colectivos con orientaciones 
sexuales muy diversas ha contribuido a la ruptura de la triada - sexualidad, reproducción y matrimonio-. Por otra parte, dentro de este incremento de la visibilidad, hay que recordar que la legislación sobre las uniones entre personas del mismo sexo se inició en España a finales de la década de los noventa, cuando, entre 1998 y 2005, doce de las diecisiete comunidades autónomas españolas fueron creando una normativa en lo que se refiere a las parejas de hecho, independientemente de la orientación sexual. Este proceso paulatino, sin duda, fue preparando el terreno para dar un paso más hasta llegar a la legislación del matrimonio homosexual, incluyendo el derecho a la adopción (Platero, 2007).

Han pasado más de diez años desde la formulación legal del matrimonio entre personas del mismo sexo en España. Se trata de un período difícil en la sociedad española, pues la crisis económica, iniciada a finales del 2007, ha tenido efectos devastadores en la destrucción de empleo y se acompañó de la puesta en marcha de un programa de recortes sociales de distinta naturaleza (sanidad, educación, dependencia, etc.). Los primeros estudios que han abordado el impacto de la crisis sobre la demografía española han revelado que éste ha sido, de momento, bastante modesto en los distintos indicadores, salvo en el paso que se ha dado de un saldo migratorio positivo a otro negativo y la consiguiente pérdida de población. No obstante, también se apunta a que se han reforzado algunas tendencias demográficas como el descenso de la fecundidad, la caída de la nupcialidad y el retraso en la edad del matrimonio, sobre todo en aquellas Comunidades Autónomas que se han visto más afectadas por el deterioro socioeconómico sobrevenido (Castro Martín et al., 2015), al mismo tiempo que han aflorado formas más plurales de conyugalidad y que se han desestigmatizado la monoparentalidad y la soltería. A modo de ilustración, la tasa de nupcialidad ha pasado de 4,8 matrimonios por cada 1000 habitantes en 2005 a 3,6 en 2015, siendo el descenso, en España, más pronunciado que el experimentado en los demás países de la UE (28).

Por tanto, una vez superado el simbólico umbral del décimo aniversario de la legalización del matrimonio entre personas del mismo sexo, parece un momento oportuno para hacer un balance y ver cuál ha sido la evolución de este tipo de evento nupcial desde su aparición, su distribución geográfica y los perfiles más comunes entre sus protagonistas. El punto de partida del presente trabajo analiza la evolución del fenómeno a lo largo de la última década, cuestiona si el matrimonio igualitario se ha ido extendiendo por la geografía española o aparece concentrado en determinados entornos, y si los perfiles sociodemográficos de sus protagonistas son más o menos heterogéneos respondiendo a un target definido. Para responder a estas preguntas se ha estructurado el artículo en tres partes.

En primer lugar, se va a examinar cuál ha sido la evolución del hecho nupcial entre parejas del mismo sexo, en estos diez años de historia. Varios análisis comparativos realizados en distintos países han puesto de manifiesto que los registros de parejas del mismo sexo pasan por varias 
etapas desde el momento en que se aprueba su legislación (ya sea bajo la fórmula de parejas de hecho o como matrimonios): en el primer año, y a menudo también en el segundo, tienen lugar un gran número de celebraciones, para a continuación producirse un retroceso sustancial, que remonta muy levemente después, momento a partir del cual se registra una estabilidad que permanece durante muchos años (Festy, 2006; Banens, 2010). La alta frecuencia en los primeros momentos de legalización se debe a la existencia de un stock de parejas que habían esperado durante mucho tiempo la aprobación de una ley para las uniones entre personas del mismo sexo. Es lo ocurrido, por el ejemplo, en el caso de Dinamarca a raíz de su ley sobre las parejas de hecho en 1989 o de Bélgica en 2001 con la legislación del matrimonio homosexual. Belliot (2013), para el caso francés, señalaba también que había que tener en cuenta la posibilidad de una dimensión numérica tímida al principio, porque numerosas parejas podían estimar más oportuno contraer matrimonio una vez que el impacto mediático y social de la ley se hubiese atenuado. Pese a ello, las primeras cifras disponibles revelan que la media mensual de matrimonios entre personas del mismo sexo fue mayor el primer año de su legislación que el segundo (Mazuyet al., 2015). Por otra parte, tenemos que tener en cuenta, como se ha apuntado anteriormente, que al igual que ha ocurrido en otros países, la ley del matrimonio entre personas del mismo sexo en España se formuló en un contexto de progresiva caída de la nupcialidad, más intensa, además, desde el inicio de la crisis económica. El propósito de este apartado es examinar si se puede hablar de que la tendencia es hacia la consolidación del matrimonio homosexual, durante este periodo, y si se aprecian o no divergencias con respecto a las parejas heterosexuales en un contexto especialmente difícil para la calidad de vida de la sociedad española por el dramático aumento del desempleo, la precarización laboral, los recortes públicos y el retroceso en multitud de políticas sociales.

En segundo lugar, el trabajo se centra en el análisis de la distribución geográfica del matrimonio entre personas del mismo sexo. En este sentido son varios los interrogantes a resolver. ¿Presenta el matrimonio de personas del mismo sexo una distribución concentrada o dispersa por la geografía española? ¿Es la concentración mayor que en el caso del matrimonio heterosexual? ¿Qué factores contribuyen a explicar su distribución? Para el caso de Estados Unidos, Black et al. (1999) hablaban hace una quincena de años de una fuerte concentración de las parejas del mismo sexo en determinadas ciudades con algunas características comunes (por ejemplo, San Francisco y Fort Lauderdale): un clima suave, estar en el litoral y ser lugares en los que tradicionalmente existía una mayor apertura o tolerancia hacia la homosexualidad. Por contra, la presencia era mucho menor en las ciudades industrializadas del medio oeste y el este como Cleveland, Buffalo o Detroit. Los autores señalaban, además, que la concentración era mayor en los sectores urbanos con mayor poder adquisitivo, lo que les llevaba a considerar también como factor explicativo la propia decisión individual, por parte de determinadas parejas, formadas por personas del mismo sexo, para instalarse en las áreas residenciales con mayor nivel de vida, aunque esta localización residencial en 
Estados Unidos, en estas grandes áreas urbanas, es más común entre los hombres gays que entre las mujeres lesbianas (Black et al, 2002). También se puede hacer alusión al caso francés, donde estudios más recientes reflejan que el matrimonio entre personas del mismo sexo está presente, sobre todo, en el litoral mediterráneo (Banens, 2016), abundando también en espacios con alto poder adquisitivo. El hábitat urbano, en general, aparece como el más propicio para vivir una homosexualidad libre. A este respecto, hay que tener en cuenta, como nos recuerda Leroy (2006), que las ciudades ofrecen mayores posibilidades de encontrar a la pareja potencial, lo que aumenta conforme el tamaño de la ciudad es más grande. Este hecho se enriquece con las migraciones internas de jóvenes homosexuales hacia espacios urbanos donde poder vivir con mayor libertad su orientación sexual. Es más, la preferencia por este tipo de ámbitos, particularmente de los hombres, se potencia más cuando se produce una apropiación de determinados espacios que ponen en marcha redes de solidaridad y de sociabilidad (Léobon, 2006). No obstante, Santos Solla (2002) introduce un punto crítico en la creación de los barrios urbanos donde se concentra la población homosexual: la revalorización urbana que conlleva excluye a los homosexuales más pobres, los cuales suelen residir en lugares como el medio rural donde la intolerancia está muy patente. Sitiados socialmente en su medio habitual, el acceso a otros espacios de más libertad no les es fácil. A unas reflexiones similares llegan Remiggi (1998) y Giraud (2013) en los que respecta al Village gai de Montreal: la recalificación urbana por el asentamiento homosexual esconde la invisibilidad de otros grupos como las lesbianas o los hombres gais con menos recursos. En lo que respecta al caso español, según Paradero (2013), en la distribución geográfica del matrimonio de personas del mismo sexo en España, tenemos que tener en cuenta variables como la densidad de población, el índice de envejecimiento y la tasa de inmigración, en el sentido de que se observa una mayor presencia en las provincias más densamente pobladas, estructuralmente más jóvenes y con mayor volumen de población extranjera.

Por último, se hace un repaso de las principales características sociodemográficas de los protagonistas del evento nupcial. En un estudio realizado, sobre las personas del mismo sexo que declararon vivir en pareja de hecho, por Cortina y Cabré (2010) a partir del Censo de 2001, se llegaba a las conclusiones siguientes: eran sobre todo jóvenes, con nivel de formación medio-alto y con residencia en espacios urbanos. El mismo estudio ponía de manifiesto un alto grado de heterogamia en función de la edad o el nivel de estudios, característica esta última que Cortina (2016) ha confirmado en un análisis sobre las primeras parejas de personas del mismo sexo que contrajeron matrimonio. Beillot (2009), en un estudio comparativo entre distintos países en los que existe legislación sobre las parejas del mismo sexo (bien como parejas de hecho o casadas), pone de manifiesto que los cónyuges formalizan la relación a edades más altas que las parejas heterosexuales. El autor se pregunta si conforme va pasando el tiempo puede haber una tendencia hacia el rejuvenecimiento de las parejas que deciden dar el paso de casarse. Tal es la tendencia 
que se ha apreciado en Reino Unido con la figura de las parejas de hecho: las uniones formadas por personas del mismo sexo que accedían a este tipo de uniones iban siendo cada vez más jóvenes conforme la antigüedad de la ley era mayor (aunque la edad seguía siendo más alta que en cónyuges heterosexuales).

\section{Metodología y fuentes}

Para responder a los anteriores interrogantes nos hemos servido de dos fuentes principales: los datos sobre matrimonios del Movimiento Natural de Población (MNP) y, de forma auxiliar, de los que proporciona el Censo de Población de 2011, fuentes ambas gestionadas por el Instituto Nacional de Estadística (INE).

La información sobre la nupcialidad en España aparece recogida (junto a la relativa a nacimientos y defunciones) en las Estadísticas del MNP del INE, aunque se elaboran en colaboración con las Comunidades Autónomas. La fuente primaria de la información son los Registros Civiles que mensualmente envían a las delegaciones del INE los boletines sobre los matrimonios que se han celebrado. A partir del tratamiento de dicha información el INE distribuye dos tipos de datos: tabulaciones cerradas con resultados detallados a nivel nacional, para provincias, capitales y ciudades mayores de 50000 habitantes; y ficheros anuales de microdatos para su explotación por parte de los usuarios. Tanto en un caso como en otro, la serialización de los datos se ve afectada por los cambios habidos en el boletín estadístico de matrimonios a lo largo del tiempo.

Los microdatos de matrimonios del MNP, que han sido nuestra principal fuente de referencia a lo largo del análisis, contienen los datos individuales de los protagonistas de un evento nupcial, convenientemente anonimizados, con el fin de preservar la confidencialidad de la información. En lo que se refiere a los matrimonios entre personas del mismo sexo, los principales datos que se pueden consultar anualmente son: el número de contrayentes, las edades de los cónyuges, el estado civil previo de los protagonistas del evento, la nacionalidad, la profesión autoasignada por los contrayentes y el nivel de estudios. Territorialmente, la información aparece desagregada a distintos niveles: provincias, municipios mayores de 10000 habitantes y municipios por tamaños poblacionales.

En el caso que nos ocupa, en el que se ha analizado el periodo que va entre 2005 y 2015, la serie se ve afectada por las variaciones producidas en 2008 en el diseño del registro estadístico suministrado por el INE. Esto ha motivado que para algunas variables (edad, nacionalidad, estado civil previo, etc.) contemos con la serie decenal completa, mientras para otras (nivel de instrucción, profesión) solo dispongamos de una serie homogénea desde 2008.

En cualquier caso, las estadísticas sobre matrimonios del INE nos han permitido: a) dibujar la línea evolutiva del matrimonio para las parejas del mismo sexo desde la fecha de su legislación hasta los 
últimos datos disponibles, tanto para las parejas compuestas por hombres como por mujeres; b) analizar su distribución geográfica en las escalas mencionadas, tanto en datos absolutos como relativos a través de la tasa bruta media anual de nupcialidad de personas del mismo sexo (número de matrimonios de personas del mismo sexo por cada 100000 habitantes); c) conocer la edad a la que han contraído matrimonio en distintos años y comprobar si se aprecia o no una tendencia hacia el rejuvenecimiento al contraer matrimonio; d) caracterizar a los protagonistas de los enlaces contraídos y conocer su perfil sociodemográfico atendiendo a datos referentes a su nacionalidad, estado civil, nivel de instrucción u ocupación.

Por su parte, el último Censo de Población (2011) realizado en España recoge, por primera vez, información sobre los matrimonios de personas del mismo sexo. Ahora bien, aquí tenemos que tener presente que este "no censo" se ha realizado a partir de un muestreo representativo, lo que conlleva dificultades para analizar a grupos pequeños, como el que nos ocupa, por problemas de representatividad. Llegados a este punto, Beillot (2009) apunta algunos problemas que podemos encontrar en los censos basados en encuestas: que algunas personas no declaren su condición sexual por una posible discriminación o por celo de su privacidad, que es común que parejas de personas del mismo sexo no vivan en la misma residencia, etc. No obstante, sin olvidar estas cautelas, la explotación del Censo de 2011 contribuye a conocer mejor los perfiles de los matrimonios del mismo sexo en España, ya que, aunque sea de forma puntual en el tiempo, nos ofrece información que las estadísticas del MNP no recogen como por ejemplo: la cuantificación de parejas de hecho existentes, lo cual nos permitirá dimensionar de forma más exacta el volumen de uniones entre personas del mismo sexo o el número de hijos de los núcleos familiares constituidos por este tipo de parejas, datos que pueden darnos pistas a la hora de caracterizar a este colectivo.

\section{Evolución del matrimonio entre personas del mismo sexo en España: un proceso social de normalización}

En un primer momento, después de su legalización a mediados de 2005, los matrimonios entre personas del mismo sexo en España estaban compuestos mayoritariamente por parejas de sexo masculino (Figura 1). En el segundo semestre de 2005, inmediatamente después de la aprobación de la ley, los matrimonios entre hombres fueron más del doble (914) que los registrados entre mujeres (355). En 2006, primer año completo de la serie estadística, las uniones conyugales entre hombres representaban tres de cada cuatro matrimonios entre personas del mismo sexo. Ahora bien, con el paso del tiempo, esta disparidad se ha ido reduciendo hasta la práctica equiparación en 2015, momento en el que los matrimonios entre hombres representaron sólo el 51,5\% de las uniones entre personas del mismo sexo. Hasta el momento, 2006 fue la fecha en que se produjeron mayor número de inscripciones de matrimonios entre varones (3000); en el caso de las mujeres, la cifra más alta se ha alcanzado en 2015 (1813). 
Figura 1. Evolución del matrimonio entre personas del mismo sexo en España, en función del género (2005-2015)

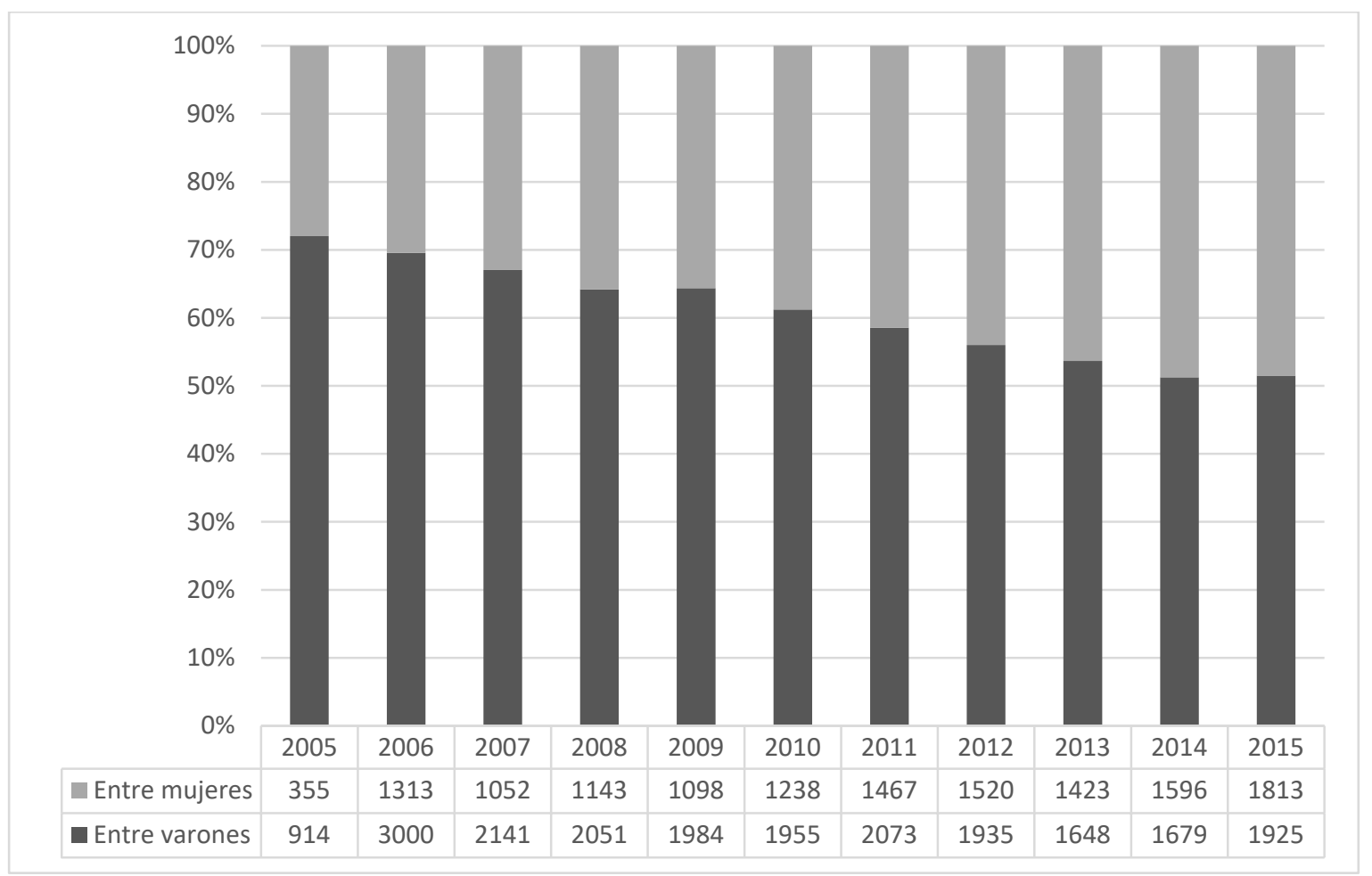

Nota: En 2005 solo se contabilizan los eventos acontecidos desde la aprobación de la ley a mediados de año.

Fuente: elaboración propia a partir de los Microdatos de matrimonios del Movimiento Natural de Población (INE, 2005-2015)

La trayectoria evolutiva del número de matrimonios (tabla de la Figura 1) ha sido diferente para los dos sexos: en el caso de los matrimonios entre hombres, la tendencia evolutiva es negativa a partir de 2007, aunque se aprecia un pequeño repunte, tanto en 2011, como al final de la serie; por lo que respecta a las uniones entre mujeres, se dibuja una línea algo titubeante al inicio, si bien a partir de 2011 se empiezan a registrar valores algo más elevados. En este sentido, cabe sopesar si la visibilidad de la orientación sexual, ya sea a través del matrimonio o con otras fórmulas de convivencia, pueda encontrar más obstáculos sociales en algunas mujeres en determinados contextos, como el rural (Goicoechea Gaona et al., 2015). Hay un elemento a poner de relieve en el caso de las mujeres: el matrimonio puede ser muy importante cuando hay un proyecto de maternidad por los derechos legales que ofrece sobre el marido de la relación previa. Gimeno (2003) habla de una triple marginalización en un contexto aún más amplio: en el seno de la sociedad gay, en la heterosexual y en el movimiento feminista. Platero (2007) subraya la necesidad de aplicar una perspectiva de género a la hora de analizar el matrimonio entre personas del mismo sexo: por la mayor invisibilidad de las mujeres lesbianas, por su posicionamiento en un segundo plano económico (trabajadoras precarias) y social, y, por tanto, con un punto de partida distinto. Sin perder de vista la pertinencia de este enfoque, hay que poner de relieve, también, que, en un 
intervalo de tiempo corto, la diferencia entre los matrimonios entre hombres y entre mujeres en términos numéricos se ha ido acortando progresivamente.

Figura 2. Evolución del matrimonio entre personas del mismo sexo, diferenciando entre géneros, y el matrimonio heterosexual $(2006=100)$

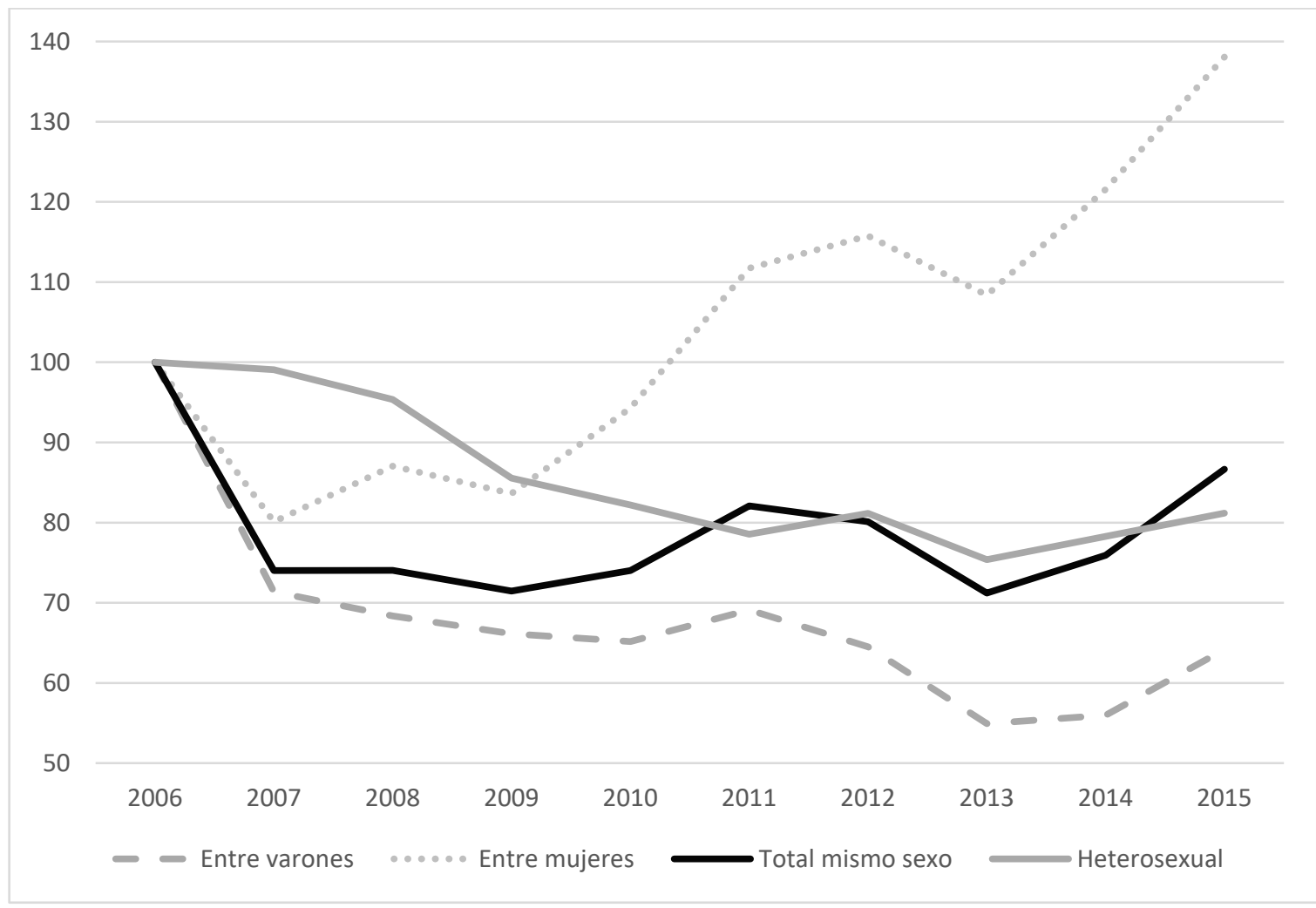

Fuente: elaboración propia a partir de los Microdatos de matrimonios del Movimiento Natural de Población (INE, 2006-2015)

Relativizando las cifras y considerando 100 el primer año completo de la serie (2006), se aprecia (Figura 2) que ese primer año es en el que se produjeron un mayor número de casamientos, dato que no se ha vuelto superar. Al año siguiente tuvo lugar la principal caída, para a partir de entonces mantenerse en una proporción similar, con una ligera subida entre 2009 y 2011, desde esos momentos se vuelve a un declive que perdura hasta el repunte final que las cifras parecen indicar. En este sentido, se puede afirmar, como se aludía en la introducción, que la cifra récord de 2006, al año siguiente de la legalización, se explica por la acumulación de un stock de parejas que no esperaron más para dar el paso y que posiblemente llevaban aguardando mucho tiempo el momento de casarse. Ahora bien, esta lectura es aplicable al total de matrimonios entre personas del mismo sexo y al caso de los matrimonios masculinos, no así al de los matrimonios femeninos. En el caso de las mujeres también se produce un descenso drástico en el segundo año completo de la serie (2007), pero a partir de 2009 se acelera su crecimiento y se supera con creces el stock 
inicial de parejas casadas al inicio de la legalización del matrimonio igualitario, observándose un crecimiento mantenido prácticamente hasta el final de la serie.

Como se dijo en la introducción, no hay que olvidar que durante la crisis económica se ha producido una caída general de la nupcialidad en España (con la salvedad de los años 2011 y 2014). Ahora bien, la secuencia ha sido diferente entre las parejas del mismo sexo y las heterosexuales pues mientras en las primeras la tendencia evolutiva ha sido más titubeante, entre las segundas los datos apuntan claramente a la baja. En lo que respecta a los matrimonios entre personas del mismo sexo, la caída más brusca tuvo lugar entre 2006 y 2007, porque lógicamente en el primer año se agruparon las celebraciones de muchas bodas de personas, mayoritariamente hombres, que no habían podido ejercer este derecho hasta ese momento. A partir de 2008 la tendencia es de nuevo al alza hasta 2011, en parte debido también, como hemos visto, al aumento progresivo de las bodas entre mujeres. Después el ritmo es similar al de las parejas heterosexuales, subiendo en el último año del que disponemos de datos.

Por lo que respecta al número de rupturas matrimoniales entre las parejas del mismo sexo, cabe apuntar que ha ido aumentando con los años. Rápidamente se han hecho lecturas muy críticas desde algunos medios conservadores aludiendo a la poca o nula sostenibilidad por naturaleza en las relaciones de pareja entre personas del mismo sexo. Ahora bien, esta evolución ascendente en el tiempo era de esperar por el propio aumento acumulativo en el número de matrimonios: aumenta el número de parejas casadas, se incrementa la probabilidad de las rupturas. Pese a ello, el nivel de rupturas considerando el stock de matrimonios y de separaciones para el periodo 2007-2014 es del 9,2\%: es decir, por cada 100 bodas hubo algo menos de 10 rupturas, apreciándose, durante este periodo, que las rupturas entre los matrimonios de personas del mismo sexo ha sido superior a la de los matrimonios heterosexuales. Pero aquí cabe tener en cuenta una cuestión, que durante los años de la crisis económica ha descendido el número de separaciones, apuntándose desde algunas perspectivas a las dificultades económicas para explicarlo: el deterioro socioeconómico ha podido desmotivar a muchas parejas para separarse por las dificultades para gestionar la ruptura y mantener dos hogares, sobre todo en los casos de familias con hijos.

Otra de las cuestiones que se ha puesto de relieve es la duración del matrimonio entre personas de mismo sexo subrayando su escasa durabilidad. Si se analizan los datos de la Estadística de Nulidades, Separaciones y Divorcios (INE, 2013-2016) para el periodo 2013-2016 se podrá comprobar que más de la mitad de los desenlaces corresponden a matrimonios con una duración de más de cinco años (54,1\%). Si añadimos las rupturas tras un periodo de convivencia de 2 a 4 años, se estaría en torno a un porcentaje del $90 \%$. Por tanto, los desenlaces de corta duración (como mucho un año de matrimonio), si bien alcanzan una proporción más alta que entre las parejas heterosexuales (por debajo del 30 \% en las mismas fechas) no dejan de ser minoritarios en 
la evaluación de conjunto. Andersson et al. (2006) en su estudio sobre el matrimonio homosexual en Suecia y Noruega comprobaron que la duración de los enlaces entre personas del mismo sexo era menor que en las parejas heterosexuales. En este sentido recordaban que había que tener en cuenta que la menor participación en la paternidad o maternidad no suponía un obstáculo a la hora de calibrar la ruptura, lo que sí podía ocurrir en los matrimonios homosexuales. Aun así, los autores no se arriesgaban a hacer un pronóstico a largo plazo debido a que, como en este caso, el estudio se realizó en los primeros años de vida de la legislación.

Por tanto, ¿qué lectura se puede hacer de estos diez primeros años de matrimonio entre personas del mismo sexo en España desde el punto de vista evolutivo? La interpretación a realizar tiene varios elementos a considerar: por una parte, habría que tener en cuenta que, en líneas generales, la tendencia que se dibuja es en cierto modo ascendente, sobre todo, si se considera que ha tenido lugar en un periodo especialmente dificultoso para España, por el aumento del paro y la política de austeridad de estos años. La evolución guarda paralelismo con lo sucedido, en líneas generales, en otros países que legalizaron con distintas fórmulas las uniones entre parejas del mismo sexo: dos primeros años con cifras récords de matrimonios registrados, que se corresponden con el stock acumulado durante años, posterior bajada y apertura de un periodo más o menos estable. En segundo lugar, es de subrayar el distinto recorrido de las líneas en función del género, con el mayor protagonismo que ha ido alcanzando el matrimonio entre mujeres hasta llegar a una proporción casi equitativa. Por último, es cierto que el número de separaciones ha ido aumentando, pero su proporción no invita a pensar en caracterizar al matrimonio entre personas del mismo sexo por una mayor fragilidad que el producido entre parejas heterosexuales. Desde algunos análisis se subraya la exigüidad de las cifras del matrimonio entre personas del mismo sexo en los primeros años (Lucas Marín, 2010), pero como subraya Pichardo (2009) la trascendencia del matrimonio homosexual en términos cuantitativos ha sido, quizás, menor que la que en principio se esperaba (al menos los primeros años), pero ha sido fundamental en cuanto a su impacto cultural, legal y político. En el análisis cualitativo que al respecto realiza este autor, los entrevistados ponían de manifiesto que el matrimonio era una cuestión de ciudadanía y de igualdad de derechos. De hecho, algunas parejas reconocieron que un argumento de peso para casarse fue su activismo por reivindicar su relación y en pro de una mayor aceptación social. De otro lado, otros entrevistados optaban por no casarse por los mismos motivos que cualquier pareja heterosexual: no compartir los valores, derechos y deberes que acompañan al matrimonio.

\section{Distribución geográfica: claves de una lectura territorial}

La distribución del matrimonio entre personas del mismo sexo en España presenta una geografía bastante desigual, cualquiera que sea la escala territorial de estudio que se tome. En un primer análisis realizado por Comunidades Autónomas, la suma de los enlaces registrados en Cataluña y la 
Comunidad de Madrid representa el 44,68 \% del stock matrimonial entre personas del mismo sexo en el periodo 2005-2015. Si añadimos Andalucía, la Comunidad Valenciana y Canarias, se estaría hablando de algo más de tres de cada cuatro matrimonios. Por tanto, cinco comunidades, independientemente del género de las parejas, engloban el 78,19\% de los matrimonios entre personas del mismo sexo. Con la excepción de Canarias, donde se registran, históricamente, indicadores más altos de progreso social (proporción de hijos de mujeres no casadas, proporción de matrimonios civiles, etc.), se trata de cuatro de las comunidades más pobladas de España. En el caso de los matrimonios heterosexuales, estas cinco comunidades agrupan un porcentaje 15 puntos menor $(63,69 \%)$, lo que revela una mayor concentración geográfica en el caso de las parejas conformadas por personas del mismo sexo.

Cuando pasamos al análisis por provincias (Figura 3), hay dos que resaltan especialmente: Barcelona y Madrid, ambas con una proporción muy similar, pues concentran el 40,77\% de los matrimonios de parejas del mismo sexo entre 2005 y 2015. Añadiendo Valencia, Alicante y Málaga, rebasamos el 56,78\%. En cualquier caso, con la salvedad de Madrid, destaca la notable incidencia del fenómeno en la España insular y el arco mediterráneo donde los valores relativos se sitúan por encima de 8 matrimonios por cada 100000 habitantes. Por lo demás, la menor incidencia tiene como escenario la mayor parte del interior peninsular, destacando especialmente por ellos Zamora, Teruel, Lugo, Soria, Orense, Cuenca, Ciudad Real o Jaén.

La distribución geográfica difiere ligeramente en función del sexo de las parejas contrayentes. Para empezar, el matrimonio entre hombres parece apuntar a una mayor concentración. Tomando los valores absolutos para el periodo 2005-2015, sólo tres provincias acogen la mitad de las uniones entre varones: Madrid, Barcelona y Valencia. Con Alicante y Málaga se alcanza el 60 \%. En lo que respecta a las mujeres, para llegar a un porcentaje similar, a dichas provincias, habría que añadir las de Illes Balears y Sevilla (60\%).

También se puede distinguir entre ámbitos en los que, dentro del matrimonio de personas del mismo sexo, predomina el que se produce entre hombres sobre el de mujeres. Ello ocurre en 45 de las 52 provincias existentes (Figura 3), pero, especialmente, en Zamora, Málaga, Madrid y Palencia, donde el porcentaje de uniones entre varones se sitúa por encima del $65 \%$ del total. Por el contrario, en los casos de Ávila, Melilla, Burgos, Ceuta, Huelva y Guadalajara el número de matrimonios entre mujeres supera al registrado entre varones. Por su parte, en Albacete, Lleida, Castellón, Guipúzcoa, Navarra, Segovia, Córdoba y Badajoz se aprecia una distribución equilibrada. 
Figura 3. Tasa bruta de nupcialidad entre parejas del mismo sexo por cada 100000 habitantes y distribución porcentual atendiendo al género (2005-2015)

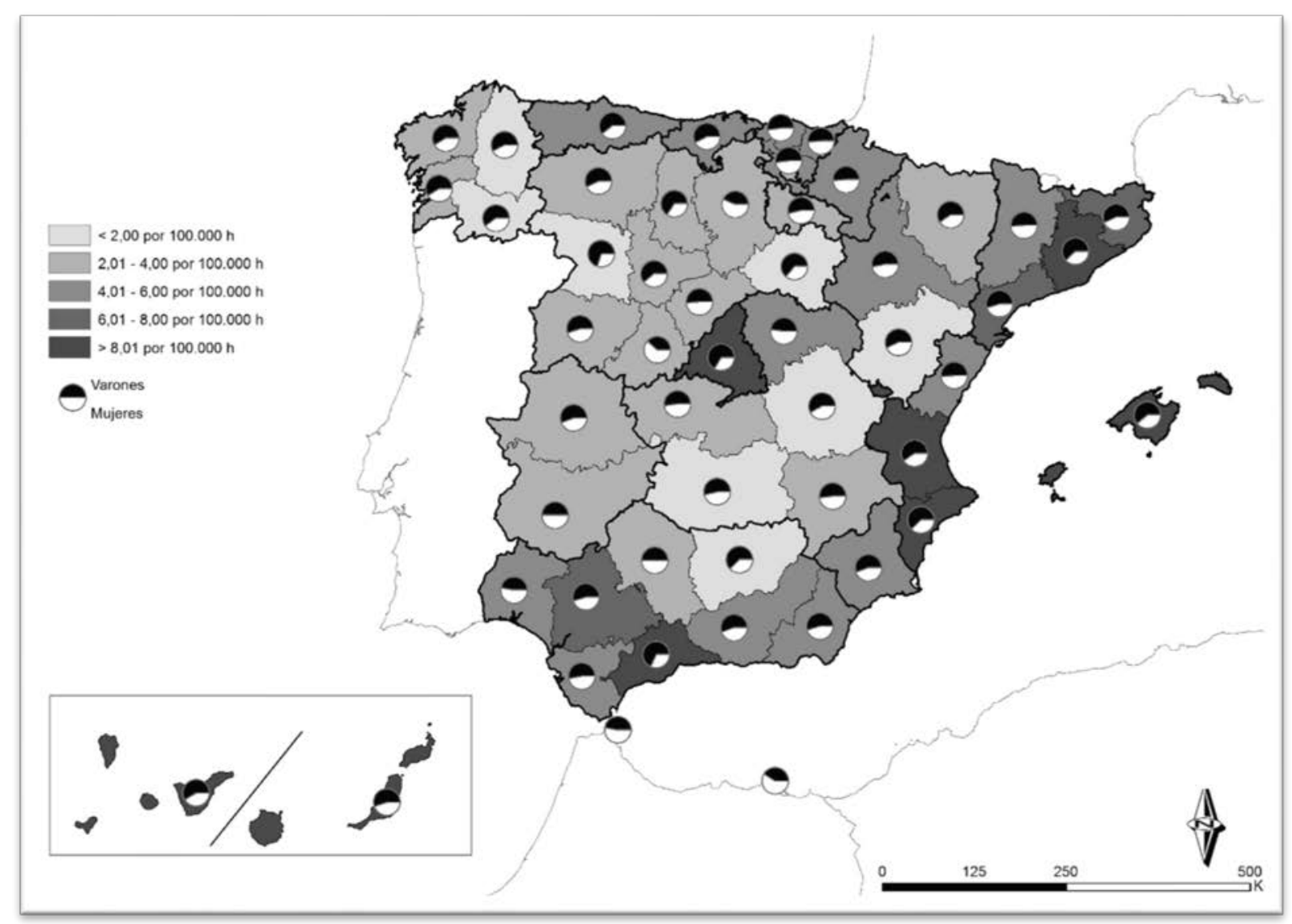

Fuente: elaboración propia a partir de los Microdatos de matrimonios del Movimiento Natural de Población (INE, 2005-2015)

Aunque los boletines matrimoniales, que sirven de base a las estadísticas del MNP, recogen, entre otra información, el municipio de inscripción del evento, y esto permitiría un análisis geográfico detallado de la distribución espacial del fenómeno, los microdatos facilitados por el INE merman considerablemente esta posibilidad ya que solo consignan la identificación del municipio cuando este cuenta con más de 10000 habitantes. Esto supone un sesgo ya que dicho umbral poblacional impide un análisis pormenorizado de la cuestión que sería de sumo interés. Si podemos conocer, sin embargo, los municipios que, superando dicho volumen poblacional, presentan mayores tasas brutas de nupcialidad entre personas del mismo sexo (Tabla 1).

Todas estas localidades, sobre todo Sitges (Barcelona), triplican con creces el promedio nacional situado en 6,90 casos por cada 100000 habitantes, al tiempo que revelan claramente la tipología de municipios preferida por el colectivo homosexual: entornos costeros cuyo sustento económico es el turismo (Sitges, Benalmádena, San Bartolomé de Tirajana, etc.) y enclaves, de marcado carácter residencial, en los entornos metropolitanos de las grandes ciudades (Villanueva de la Cañada, Aranjuez, Pallejá, etc.). 
Tabla 1. Municipios de más de 10000 habitantes con mayores tasas brutas de nupcialidad entre personas del mismo sexo (casos por cada 100000 habitantes)

\begin{tabular}{|l|l|c|l|l|c|}
\hline \multicolumn{1}{|c|}{ MUNICIPIO } & \multicolumn{1}{|c|}{ PROVINCIA } & TBNup & \multicolumn{1}{c|}{ MUNICIPIO } & PROVINCIA & TBNup \\
\hline Sitges & Barcelona & 134,39 & Torremolinos & Málaga & 23,74 \\
\hline $\begin{array}{l}\text { Villanueva de la } \\
\text { Cañada }\end{array}$ & Madrid & 48,67 & Argentona & Barcelona & 23,39 \\
\hline Mongat & Barcelona & 43,02 & Aranjuez & Madrid & 22,60 \\
\hline El Escorial & Madrid & 29,59 & La Seu d'Urgell & Lleida & 21,63 \\
\hline Benalmádena & Málaga & 29,47 & Arenys de Mar & Barcelona & 21,46 \\
\hline $\begin{array}{l}\text { Sant Pere de } \\
\text { Ribas }\end{array}$ & Barcelona & 28,64 & $\begin{array}{l}\text { Corberá de } \\
\text { Llobregat }\end{array}$ & Barcelona & 20,53 \\
\hline $\begin{array}{l}\text { San Bartolomé de } \\
\text { Tirajana }\end{array}$ & $\begin{array}{l}\text { Las Palmas de } \\
\text { Gran Canaria }\end{array}$ & 27,36 & $\begin{array}{l}\text { San Agustín de } \\
\text { Guadalix }\end{array}$ & Madrid & 20,50 \\
\hline Pallejá & Barcelona & 25,64 & $\begin{array}{l}\text { Sant Just } \\
\text { Desvern }\end{array}$ & Barcelona & 20,41 \\
\hline Puerto de la Cruz & $\begin{array}{l}\text { Santa Cruz de } \\
\text { Tenerife }\end{array}$ & 25,58 & Santa Brígida & $\begin{array}{l}\text { Las Palmas de } \\
\text { Gran Canaria }\end{array}$ & 20,22 \\
\hline Santa Pola & Alicante & 23,81 & & & \\
\hline
\end{tabular}

Fuente: elaboración propia a partir de los Microdatos de matrimonios del Movimiento Natural de Población (INE, 2005-2015)

Los datos ocultan, sin embargo, la incidencia del fenómeno nupcial entre personas del mismo sexo en los municipios de menor entidad poblacional para los que solo se dispondría de un dato global calculado a partir de la agrupación de eventos por tamaños poblacionales de los municipios (Tabla 2)

Tabla 2. Tasas brutas de nupcialidad entre personas del mismo sexo por tamaños poblacionales de los municipios (casos por cada 100000 habitantes)

\begin{tabular}{|c|c|c|c|c|c|c|}
\hline$<10000 \mathrm{~h}$ & $\begin{array}{c}10000- \\
20000 \mathrm{~h}\end{array}$ & $\begin{array}{c}20000- \\
50000 \mathrm{~h}\end{array}$ & $\begin{array}{c}50000- \\
100000 \mathrm{~h}\end{array}$ & $\begin{array}{c}>100000 \mathrm{~h} \\
\text { no capitales }\end{array}$ & $\begin{array}{c}\text { Capitales de } \\
\text { provincia }\end{array}$ & Total nacional \\
\hline 6,31 & 6,85 & 6,95 & 6,92 & 5,07 & 7,85 & 6,90 \\
\hline
\end{tabular}

Fuente: elaboración propia a partir de los Microdatos de matrimonios del Movimiento Natural de Población (INE, 2005-2015)

Las tasas parecen apuntar a que, conforme aumenta el tamaño poblacional, mayor es la incidencia de fenómeno, tendencia que no se cumple en el caso de los municipios mayores de 100000 habitantes que no son capitales de provincia, pues estos presentan una incidencia mucho más baja que el promedio nacional. Las capitales provinciales, sin embargo, se destacan como las áreas geográficas con valores más elevados.

En este sentido, los orígenes urbanos de los protagonistas de enlaces entre personas del mismo sexo son absolutamente predominantes, tanto entre hombres como entre consortes mujeres. El porcentaje de nacidos en ciudades de más de 100000 habitantes para las parejas de sexo femenino, según el Censo de 2011, es de cerca del $50 \%$ y de un $38 \%$ en el caso de los 
hombres, lo que vendría a subrayar la hipótesis de que la homosexualidad femenina supone un mayor desafío social en el medio rural.

Huyendo de la dispersión que supondría un análisis para los municipios mayores de 10000 habitantes, una forma de unificar el estudio desde el punto de vista territorial sería abordar la incidencia del matrimonio entre personas del mismo sexo calculando las tasas brutas de nupcialidad en cada provincia diferenciando entre la capital y el resto provincial. Si establecemos tres niveles de incidencia: elevado, si las tasas superan los 7 casos por cada 100000 hab., medio, si se sitúan entre el 4 y el 6 por cada 100000 hab. y bajo, si no alcanzan el 4 por cada 100000 hab. se puede establecer una tipología (Figura 4) diferenciando las provincias según la incidencia del fenómeno en la capital y en el resto de su territorio.

Figura 4. Tipología provincial según la incidencia del matrimonio entre personas del mismo sexo, en capitales y resto de provincia (2005-2015)

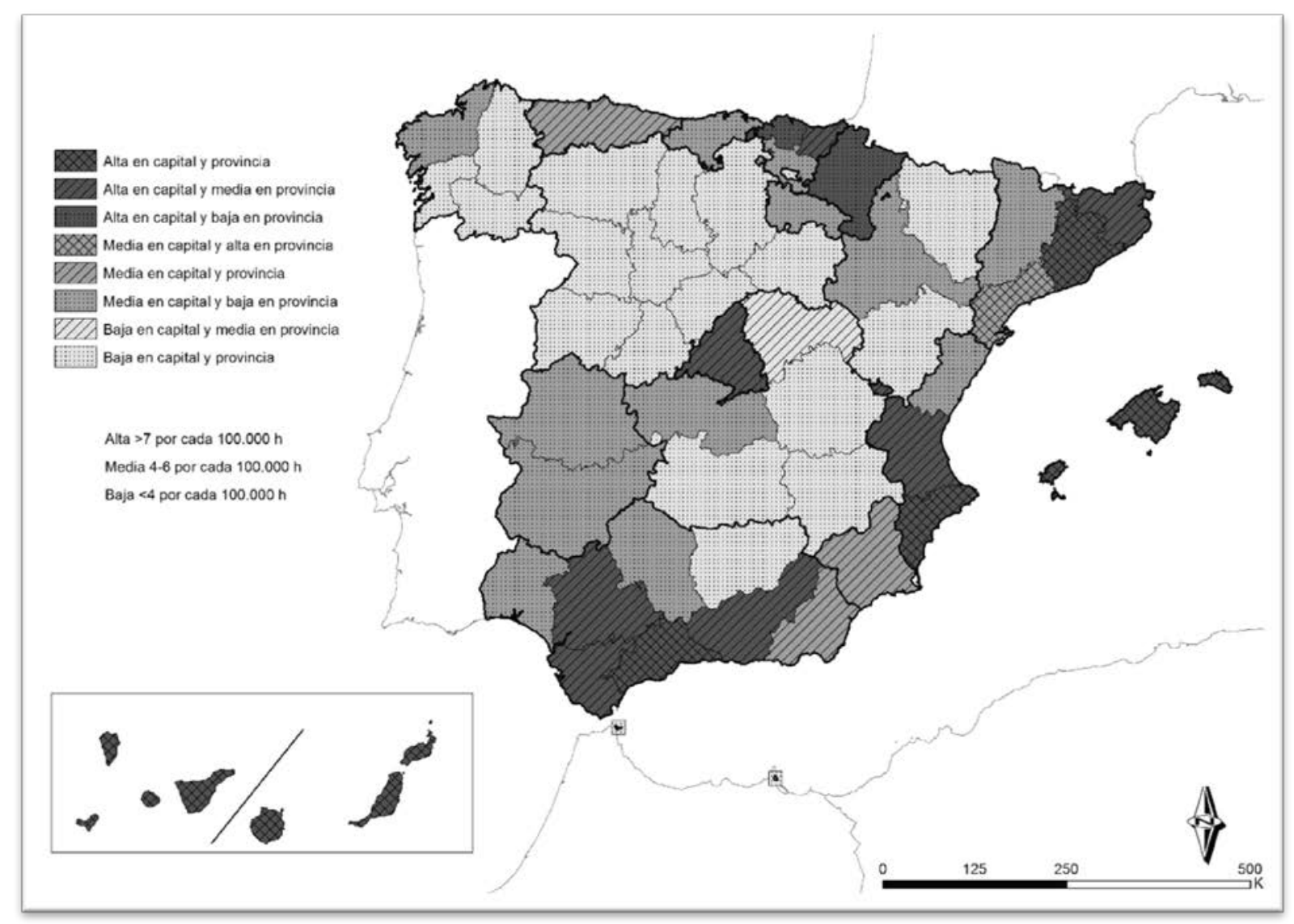

Fuente: elaboración propia a partir de los Microdatos de matrimonios del Movimiento Natural de Población (INE, 2005-2015)

Tendríamos así provincias con alta incidencia del fenómeno, tanto en la capital, como en el resto de la provincia, denotando un amplio calado y la existencia de sociedades tolerantes y abiertas. Esto ocurre en los dos archipiélagos, Barcelona, Málaga y Alicante. Como provincias con alta incidencia 
en la capital, pero valores medios en el resto del territorio, estarían Madrid, Gerona, Guipúzcoa, Valencia, Cádiz, Sevilla y Granada, mientras que con una alta incidencia en la capital provincial, pero baja en el resto, estarían Vizcaya y Navarra.

Con valores medios (de entre 4 y 6 eventos por cada 100000 hab.), en la capital y altos(por encima de 7 enlaces matrimoniales por cada 100000 hab.), en el resto de la provincia, estaría únicamente el caso de Tarragona. Valores medios tanto en la capital como en el resto provincial se registran en Asturias, Murcia y Almería, mientras que capitales con valores bajos y restos provinciales con niveles medios son un poco más habituales: A Coruña, Cantabria, Álava, La Rioja, Zaragoza, Lleida, Castellón, Toledo, las dos provincias extremeñas, Huelva y Córdoba.

Si exceptuamos Guadalajara, que se caracteriza por valores bajos en la capital y altos en la provincia, el resto del territorio: todas las provincias de Castilla-León, casi toda Castilla-La Mancha (menos Toledo y Guadalajara), Galicia (menos A Coruña), Aragón (menos Zaragoza), Jaén y las ciudades autónomas de Ceuta y Melilla se caracterizan por niveles bajos tanto en las cabeceras, como en el resto de los territorios provinciales.

La distribución del matrimonio entre parejas del mismo sexo, sobre todo en lo que se refiere a los enlaces entre varones, guarda gran paralelismo con las provincias y ciudades que presentan un grado más alto de visibilidad de la comunidad homosexual. Fernández Salinas (2008) en un estudio basado en los espacios de frecuentación entre personas del mismo sexo (para desarrollar actividades de sociabilidad, realizar compras o contratar servicios, etc.) sitúa a España como uno de los países del mundo con una visibilidad más nítida. Ahora bien, el autor también pone de manifiesto fuertes contrastes geográficos en el interior de España: las referencias se concentran sobremanera en los mayores centros urbanos del país y sus principales enclaves turísticos, sobre todo en el Mediterráneo y los dos archipiélagos (Fernández Salinas, 2007a). Por el contrario, con la salvedad de Madrid y en menor medida de Sevilla y Zaragoza, el interior de España se caracteriza por una visibilidad de la comunidad homosexual reducida. Estos contrastes territoriales se extienden a nivel de Comunidades Autónomas y en gran medida con la distribución del matrimonio homosexual (Fernández Salinas, 2007b): la visibilidad reducida se corresponde con el centro del país (las dos Castillas, Extremadura y La Rioja) y del norte (Galicia, Asturias, Cantabria y Navarra).

Otro elemento que tenemos que tener en cuenta es el desarrollo turístico y especialmente aquellos lugares en España que constituyen destinos preferentes para personas homosexuales. Como pone de relieve Kanai (2014) en las últimas décadas la diversidad sexual ha pasado a constituir una variable que algunas ciudades han desarrollado en un contexto de competitividad para atraer a turistas homosexuales. Ciertos estudios plantean críticamente algunas de estas estrategias de marketing como por ejemplo el llevado a cabo por Manchester (Hughes, 2010). En lo que respecta 
al caso español, García Escalona (2000) distingue dos tipos de espacios: por una parte, las grandes ciudades, en las que algunas de sus áreas se han consolidado como enclaves gays conocidos (por ejemplo, Chueca en Madrid o el Eixemple, en Barcelona); y por otra parte, espacios denominados "satélites" en forma de resorts para gays y lesbianas situados, sobre todo, en ciudades medias del arco Mediterráneo (Sitges, Mongat o Sant Pere de Ribes en Cataluña o Torremolinos y Benalmádena en Andalucía), y la España insular, caso de Gran Canaria, uno de los principales destinos gays en Europa (Melián González et al., 2011), por ser un enclave que reúne algunos de los recursos más valorados por la comunidad homosexual: un clima cálido, que haya una escena gay asentada y un medio social que no se muestre hostil. Vemos, pues, que estos destinos turísticos gays españoles también guardan gran paralelismo con la distribución del matrimonio entre personas del mismo sexo que se revela en el presente análisis.

De la bibliografía manejada se desprende que hay cuatro factores que inciden en la desigual distribución geográfica del matrimonio entre personas del mismo sexo: el nivel de renta, ya que parece ser que conforme éste aumenta también lo hace matrimonialidad entre parejas del mismo sexo; el grado de urbanización pues el matrimonio entre personas del mismo sexo es más frecuente en áreas urbanas que en zonas rurales, ya que en las primeras parece haber un mayor grado de apertura hacia este tipo de uniones; el índice de envejecimiento, puesto que en espacios más jóvenes es más factible encontrar un clima de tolerancia ante las uniones entre personas del mismo sexo; y la tasa de extranjería, debido, como se verá más adelante, al elevado número de matrimonios mixtos en el caso de las uniones entre personas del mismo sexo. Al correlacionar dichas variables con la incidencia del matrimonio entre personas del mismo sexo a escala provincial (Tabla 3), tres de ellas, parecen estar relacionadas, si bien las correlaciones no son muy fuertes (el coeficiente de Pearson se sitúa siempre por debajo de $+/-0,7$, umbral a partir del cual se considera que existe una notable correspondencia entre dos variables). Así, las tasas de nupcialidad entre personas del mismo sexo aumentan conforme lo hacen los niveles de urbanización y de extranjería. Por el contrario, disminuyen cuando el índice de envejecimiento aumenta. Solo en lo que respecta al nivel de renta la correlación es prácticamente inexistente, quizá por lo grosero de la escala de análisis.

Centrándonos en los cuatro indicadores que muestran cierto grado de correlación se procedió a un análisis multivariable con objeto de obtener una tipificación provincial, para ello se aplicó una Clasificación Ascendente Jerárquica (en adelante CAJ), análisis para el que Philcarto, que fue el software utilizado, emplea la adaptación de un algoritmo original de Jambu y Lebeaux (1983) que actúa en dos etapas y que reduce considerablemente el tiempo para establecer jerarquías.

La CAJ, como todas las técnicas cluster, lo que hace es generar una distancia entre casos y agrupar éstos en función de la mencionada distancia, ateniéndose, eso sí, a la máxima de conservar la mayor homogeneidad interna entre los elementos de un grupo y mantener la mayor diferenciación 
posible entre grupos. Dicho de forma un poco más técnica, se persigue una minimización de la varianza intraclase y una maximización de la varianza interclases.

Tabla 3. Coeficiente de Pearson entre la incidencia del matrimonio entre personas del mismo sexo a nivel provincial y otras variables

\begin{tabular}{|l|r|r|r|r|r|}
\hline \multicolumn{1}{|c|}{ Variables } & $\begin{array}{c}\text { Tasa bruta de } \\
\text { nupcialidad. } \\
\text { Mismo sexo } \\
\text { (2014) }\end{array}$ & \multicolumn{1}{|c|}{ Nivel de } \\
renta(2011) & $\begin{array}{r}\text { \% Pob. } \\
\text { urbana } \\
\text { (2014) }\end{array}$ & $\begin{array}{c}\text { \%>65 años } \\
\text { (2015) }\end{array}$ & $\begin{array}{c}\text { \% Pob. extranjera } \\
\text { (2015) }\end{array}$ \\
\hline $\begin{array}{l}\text { Tasa bruta de } \\
\text { nupcialidad. Mismo sexo } \\
\text { (2014) }\end{array}$ & 1 & 0,091 & 0,455 & $-0,458$ & 0,422 \\
\hline Nivel de renta (2011) & 0,091 & 1 & 0,181 & 0,128 & 0,204 \\
\hline \% Pob. urbana (2014) & 0,455 & 0,181 & 1 & $-0,473$ & 0,178 \\
\hline \% 85 años (2015) & $-0,458$ & 0,128 & $-0,473$ & 1 & $-0,482$ \\
\hline $\begin{array}{l}\text { \% Pob. extranjera } \\
\text { (2015) }\end{array}$ & 0,422 & 0,204 & 0,178 & $-0,482$ & 1 \\
\hline
\end{tabular}

Fuente: elaboración propia a partir de Movimiento Natural de la Población (INE, 2014), Contabilidad Regional de España. Base 2008. Principales resultados, 2008-2013 (INE, 2014), Padrón Municipal de Habitantes (INE, 2015) y Atlas Digital de las Áreas Urbanas (Ministerio de Fomento, 2015)

En este caso, la CAJ comienza con la partición más fina posible en la que cada provincia constituye una clase, continúa con una serie de agregaciones progresivas que van agrupando las provincias estadísticamente más cercanas en cada vez en menos clases hasta culminar en una sola clase que las engloba a todas. Al mismo tiempo, se va conformando un árbol o dendograma, que al ser cortado en un determinado punto, que decide el usuario, da como resultado el número de clases de una partición. Tanteando las distintas posibilidades, en esta ocasión, se optó por el establecimiento de 6 clases situándose la altura de corte, en el dendograma, próxima al índice 0,25 que supone el 75,99\% de la inercia total, lo que quiere decir que esta partición explica casi el $76 \%$ de la varianza entre grupos.

El resultado fue el mapa de la Figura 5 que incluye un gráfico con el perfil de las tipologías establecidas y los componentes que las definen. El cartograma con la distribución geográfica de las agrupaciones define distintos patrones espacio-territoriales: el primero de ellos caracteriza a provincias con baja incidencia de matrimonio entre personas del mismo sexo, cierto protagonismo urbano, escaso envejecimiento y poca presencia de extranjeros, perfil al que responden la mayoría de las provincias de la cornisa cantábrica (Asturias, Cantabria, Álava, Guipúzcoa y Vizcaya), el alto Ebro (Navarra, La Rioja, Zaragoza), de forma más aislada Valladolid y en el sur las provincias de Sevilla, Cádiz y Granada. 
El segundo tipo, que con 15 provincias es el más numeroso, aparece caracterizado por una baja incidencia del matrimonio entre personas del mismo sexo, baja presencia de extranjeros, escasa urbanización y cierto grado de envejecimiento. Conforma un espacio bastante compacto conformado por las provincias surorientales de Castilla-León (Ávila, Segovia, Soria, Burgos), las provincias aragonesas de Huesca y Teruel, las dos provincias extremeñas, buena parte del territorio castellano-manchego (Toledo, Cuenca, Ciudad Real y Albacete) y las provincias andaluzas de Huelva, Córdoba y Jaén.

Figura 5. Tipología provincial del matrimonio entre personas del mismo sexo en España

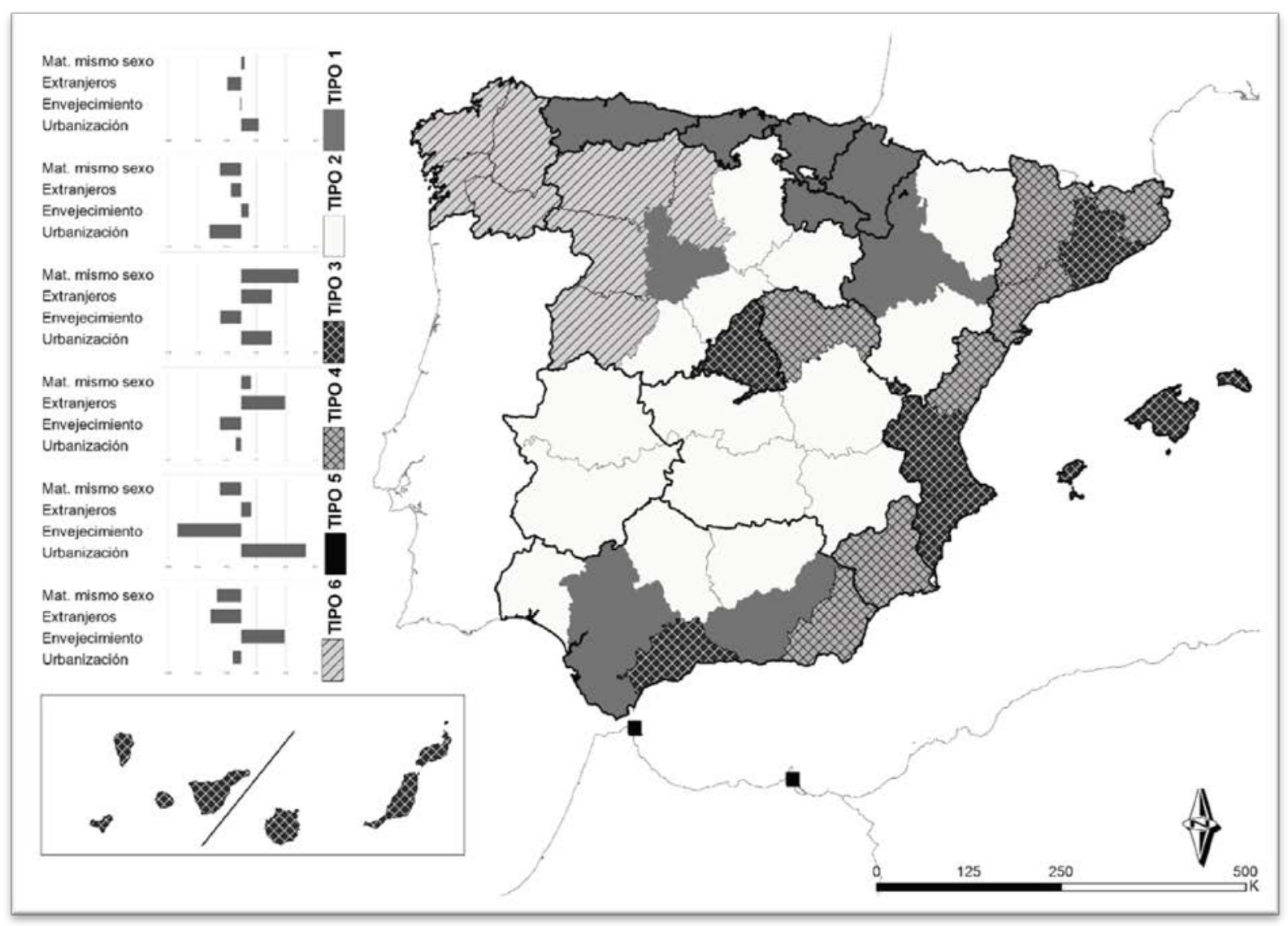

Fuente: elaboración propia a partir de los Microdatos de matrimonios del Movimiento Natural de Población (INE, 2005-2015)

La tercera tipología engloba a las provincias donde mayor presencia tiene el matrimonio entre personas del mismo sexo caracterizadas por elevados niveles de urbanización, fuerte presencia extranjera y un escaso nivel de envejecimiento. Es el caso de los archipiélagos canario y balear, Barcelona, Madrid y las provincias levantinas de Valencia y Alicante.

El cuarto tipo está conformado por el resto de provincias del arco mediterráneo (Almería, Murcia, Castellón, Tarragona y Gerona) y dos provincias de interior Lleida y Guadalajara. Se caracteriza por 
una moderada incidencia del matrimonio entre personas del mismo sexo, cierto envejecimiento, una modesta presencia urbana y un elevado protagonismo de la población extranjera.

El quinto conglomerado tiene más que nada una presencia testimonial ya que engloba a las ciudades de Ceuta y Melilla, enclaves netamente urbanos donde la incidencia del matrimonio entre personas del mismo sexo es mínima, a la par que existe una fuerte presencia extrajera y un escaso nivel de envejecimiento.

El sexto y último conjunto es el que forman las provincias del cuadrante noroccidental de la Península (A Coruña, Lugo, Orense, Pontevedra, León, Zamora, Palencia y Salamanca), básicamente el perfil es el mismo del tipo 2 con la única diferencia de que el nivel de envejecimiento es mayor.

\section{Perfiles de los contrayentes: "intrahistoria" del matrimonio entre personas del mismo sexo}

Después de observar que el matrimonio entre personas del mismo sexo es una realidad que sobresale especialmente en determinadas partes de la geografía española, es el turno ahora de ver si se caracteriza por su diversificación social o, por el contrario, presenta unos perfiles sociales más precisos. El análisis geográfico anterior ya nos ha proporcionado algunas pistas: la preferente localización en áreas urbanas con bajos niveles de envejecimiento y alta presencia de extranjeros. Como se ha visto anteriormente, la incidencia del matrimonio entre personas del mismo sexo está bastante extendida en algunos de los destinos gays españoles más conocidos. Para dibujar estos perfiles se han utilizado dos fuentes: por una parte, los microdatos del Movimiento Natural de Población correspondientes a matrimonios, con los que podemos conocer algunas características sociodemográficas en el momento en el que se produjeron los enlaces matrimoniales. Por otra, el Censo de Población de 2011, el cual, pese a sus limitaciones, nos permite completar el retrato del matrimonio entre personas del mismo sexo, eso sí,de forma puntual, en la fecha censal.

Los microdatos del MNP sobre matrimonios, para el periodo 2005-2015, apuntan a que los protagonistas de enlaces matrimoniales con personas del mismo sexo conforman un colectivo joven, pero en su primera madurez, pues la edad media de los contrayentes se sitúa en torno a los 39,5 años, seis años más que el promedio de edad en parejas heterosexuales (33,4 años). El contraste se da también en la edad media de las uniones entre hombres y entre mujeres: 40,63 años y 37,5 años, respectivamente. Las frecuencias más altas, en los dos casos, se concentran en las cohortes que van de 30 a 49 años, aunque en mayor proporción para las mujeres. De nuevo aquí hay que considerar que, posiblemente, en los primeros años contrajeron matrimonio parejas con una larga trayectoria de convivencia que, sencillamente, antes no tenían la posibilidad de casarse, lo que explica que estén infra-representadas las personas de menos de 30 años. 
En lo que respecta a una posible variación en las edades en los matrimonios entre personas del mismo sexo, conforme nos apartamos de la fecha de legalización, los datos (Figura 6) muestran que para los dos sexos la edad era mayor en los dos primeros años que en la actualidad, por encima de los 43para los hombres y en torno a los 40 para las mujeres. Esto confirma lo que venimos diciendo: en un primer momento se condensaron las uniones matrimoniales con una larga trayectoria de personas que convivían como pareja y, por tanto, con cierto grado de madurez. Aunque en el caso de las mujeres la edad matrimonial nunca más vuelve a situarse por encima de los cuarenta y la tendencia, con algún que otro titubeo, es claramente a la baja, en el caso de los varones, después de un considerable retroceso en la segunda mitad de la década pasada, a partir de 2012, se produce un significativo repunte superándose de nuevo entre los contrayentes el umbral de la cuarentena.

Figura 6. Evolución de la edad matrimonial entre personas del mismo sexo en España (2005-2015)

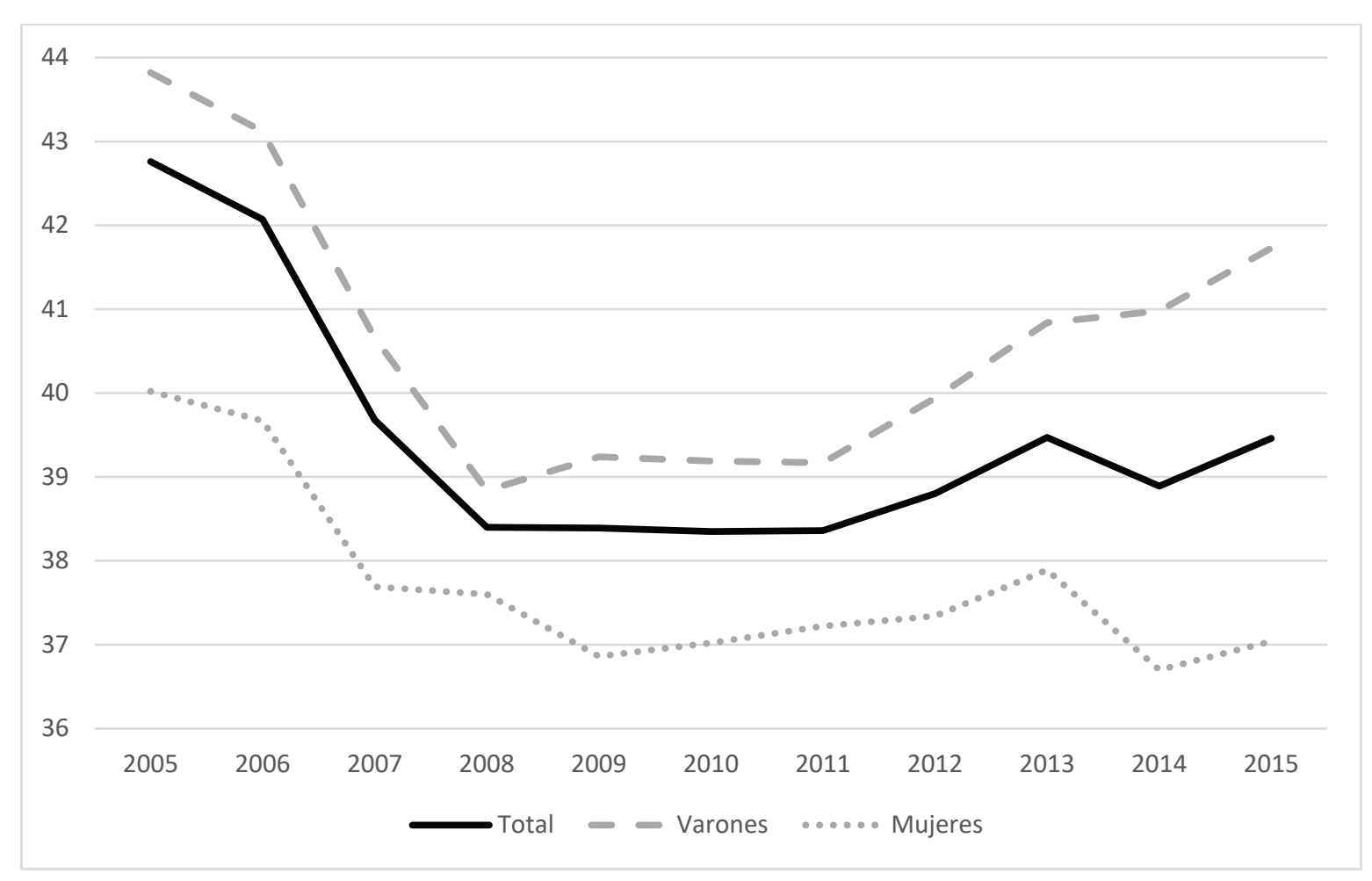

Fuente: elaboración propia a partir de los Microdatos de matrimonios del Movimiento Natural de Población (INE, 2005-2015)

Otro aspecto a destacar es la diferencia de edad dentro de la pareja: menor entre los matrimonios heterosexuales que entre los de personas del mismo sexo. Al respecto las cifras ponen de manifiesto que, mientras el 43,59\% de los matrimonios heterosexuales se producen entre personas en el mismo intervalo de edad, el porcentaje baja al 30,06\% entre los matrimonios del mismo sexo. Dentro de estos últimos, las diferencias entre matrimonios entre varones $(26,34 \%)$ y féminas 
$(35,03 \%)$ es también sustanciosa apuntando a una mayor diferencia de edad en los matrimonios masculinos. En este sentido, los microdatos apuntan a que el 20,61 \% de las parejas masculinas tienen una diferencia de edad superior a los 10 años, porcentaje que en el caso del matrimonio entre mujeres no llega al 7,68 \% y en el matrimonio heterosexual apenas rebasa el $5 \%$.

En cuanto al nivel de estudios, los matrimonios entre personas del mismo sexo están compuestos en gran proporción por cónyuges con un buen nivel de formación, según los microdatos del MNP, en el momento del enlace el 60,16\% de los casados tenían al menos estudios secundarios (Bachillerato o superior), proporción que en el caso de matrimonios heterosexuales apenas llegaba al $55 \%$. En esta ocasión la diferenciación entre los primeros, atendiendo al género, apenas es significativa: $59,84 \%$ en el caso del matrimonio entre varones y 60,59 \% en el de mujeres.

Si tomamos como umbral los estudios universitarios: 29,33 de cada 100 contrayentes en parejas del mismo sexo cuentan con titulación superior, frente a 27,26 en el caso de parejas heterosexuales. También en esta ocasión se aprecia una pequeña diferencia en el primer tipo de matrimonios si se distingue entre enlaces entre varones $(28,87 \%)$ y entre mujeres $(29,96 \%)$, pudiéndose apreciar, tanto en este caso como en el anterior, que el nivel formativo de las féminas es algo superior al de los varones.

Respecto a la actividad, los microdatos del MNP recogen la profesión autoasignada por los contrayentes al completar el boletín matrimonial. Analizando los datos comprobamos que, en torno a un $16,87 \%$ de los que contraen matrimonio con otra persona del mismo sexo, no consignan esta información, siendo éste un porcentaje similar al de las personas de matrimonios heterosexuales. Cabe pensar, a tenor del reducido número de personas que dice estar en paro, que la mayoría de quienes no rellenan este epígrafe del formulario estén desempleados.

Por lo demás, las respuestas consignadas dan para diferenciar entre lo que sería población activa (ocupados y parados) e inactiva (pensionistas, estudiantes y sus labores), situándose las respectivas proporciones, respecto al total de personas que contrajeron matrimonio entre 2005 y 2015, en el $76,22 \%$ y el 6,92\%, respectivamente, en el caso de los miembros de parejas del mismo sexo, y en el $77,83 \%$ y el $6 \%$, en el de parejas de sexo diferente. En lo que respecta al paro, los valores son igualmente próximos 6,95 \% y 6,46 \%.

Centrándonos en la población ocupada, 69,27\% entre los miembros de parejas del mismo sexo y 71,38\% entre heterosexuales y atendiendo a las categorías de la CNO11 cabe mencionar mayor presencia del colectivo homosexual que del heterosexual entre los trabajadores de los servicios de restauración y una infrarrepresentación de los primeros entre artesanos y trabajadores cualificados de las industrias manufactureras, la construcción y la minería, y operadores de instalaciones y 
maquinaria. Las diferencias de género entre las parejas del mismo sexo relativas a la actividad apenas son reseñables

Los microdatos del MNP permiten también conocer el estado civil desde el que se accede al matrimonio, en la mayoría de los casos este paso se produce desde la soltería, siendo este un hecho mucho más frecuente entre parejas del mismo sexo, 90,52\% que en el de en el de parejas heterosexuales, 87,07\%. En el caso de las primeras, si el matrimonio es entre varones, el 91,98\% son solteros, mientras que si es entre mujeres el porcentaje se queda en el 88,32\%. Si el estado civil es distinto a la soltería lo más frecuente es que se acceda al matrimonio tras un divorcio ya que la viudedad apenas propicia nuevos enlaces.

Como se ha adelantado anteriormente, un dato sorprendente de los matrimonios entre personas del mismo sexo en España es el alto porcentaje de uniones mixtas, atendiendo a la nacionalidad. Así, según la información ofrecida por los microdatos del MNP, el 30,44\% de las parejas están compuestas por una persona de nacionalidad española y otra extranjera, a las cuales se añade el que un 6,53\% de las uniones están formadas por dos extranjeros. Por tanto, las parejas compuestas por dos miembros de nacionalidad española quedan reducidas a un 63,03\%. Estos datos difieren de los registrados para parejas heterosexuales entre las que el 68,42\% de las uniones son entre españoles, el 13,94\% son matrimonios mixtos y el 17,64 \% son casamientos entre extranjeros.

Dentro del matrimonio entre parejas del mismo sexo, las diferencias entre géneros son bastante significativas pues, mientras entre las mujeres el 87,08 de las uniones son entre españolas, en el caso de los varones el porcentaje desciende al 69,53. Ello quiere decir que entre los hombres se producen más matrimonios mixtos 25,36\% y entre extranjeros 5,11\%, que entre las féminas $10,29 \%$ y $2,63 \%$, respectivamente.

Según Cortina (2013), la alta participación de personas con nacionalidad extranjera en los matrimonios entre personas del mismo sexo en España se explica, por un lado, por la masiva llegada de inmigrantes durante la pasada década (recordemos que el destino español fue el segundo más importante en cuanto a volumen en las migraciones internacionales, después de Estados Unidos) y, por otro, porque España pudo constituir durante ese periodo un punto de atracción para otros países justo a causa de la legislación que permitía los matrimonios entre personas del mismo sexo. También se podría considerar que este porcentaje alto de uniones mixtas se explica porque el matrimonio permite la estabilidad legislativa para el cónyuge extranjero (Pichardo, 2009). Otros factores a tener en cuenta son: el denominado "turismo residencial" compuesto por matrimonios homosexuales que eligen España como destino por sus condiciones climáticas, principalmente lugares como la Costa del Sol; Alicante o las Islas Canarias; el papel de las redes sociales que conectan a personas de distintos orígenes; el crecimiento de algunas 
ciudades españolas especializadas en el turismo homosexual eminentemente masculino (Torremolinos, Sitges, Madrid, San Bartolomé de Tirajana, etc.).

Otro dato interesante, según los microdatos del MNP para el periodo 2005-2015, es el algo grado de homogamia entre los contrayentes de matrimonios entre personas del mismo sexo, hecho que ponen de manifiesto datos como que el 30,06\% de las uniones se produce entre personas que comparten el mismo intervalo de edad; el 40,68 \% compartían un mismo nivel de instrucción; el 42,5\% presentaban una situación profesional similar; y por último, en su mayoría, ambos miembros partían de una misma situación civil similar al contraer matrimonio: el 85,5\% estaban solteros.

Aunque sea de forma puntual en el tiempo, el Censo de Población de 2011, permite indagar en algunas cuestiones más que nos ayudan a completar el perfil de los protagonistas de enlaces entre personas del mismo sexo. La primera de ellas tiene que ver con la significación del hecho matrimonial en el conjunto de las uniones entre personas del mismo sexo. En este sentido, el censo nos permite diferenciar entre parejas de derecho (matrimonios) y parejas de hecho. Al respecto las cifras apuntan a que mientras entre los matrimonios entre personas del mismo sexo el porcentaje de parejas de hecho apenas supone el $45 \%$, entre las parejas heterosexuales el porcentaje asciende al 85,66\%. También, atendiendo al género, dentro del matrimonio entre personas del mismo sexo, hay diferencias pues mientras entre los varones son de derecho el $46 \%$ de las uniones, entre las mujeres el porcentaje solo se sitúa algo por encima del $41 \%$. En cualquier caso, parece claro que la formalización de una relación entre personas del mismo sexo a través del matrimonio es un paso que no todo el mundo da pues son mayoría quienes se mantienen como pareja de hecho.

Otro dato de interés que nos aporta el Censo es el relativo al número de hijos de las parejas de derecho. En el caso de las del mismo sexo, el 75,79 \% no tienen hijos, con una gran diferencia entre las parejas de hombres, donde el porcentaje sube al 83,35\% y mujeres donde tal situación ocurre en el 58,98\% de los casos. Como es lógico pensar, la diferencia con las parejas heterosexuales es abismal pues solo el 23,24 \% no tienen descendencia.

En definitiva, se podría concluir diciendo que existen algunas divergencias entre las características sociodemográficas entre las personas del mismo sexo que han contraído matrimonio en este periodo y las heterosexuales. Por una parte, las parejas homosexuales presentaban una edad superior, especialmente las compuestas por varones, cuando se produjo el enlace. El nivel de instrucción medio también es mayor, aunque cabe señalar que sin un gran margen diferencial. Los matrimonios mixtos son también más numerosos, especialmente entre los enlaces entre hombres. Por el contrario, en lo referente al estatus profesional no se aprecian grandes divergencias entre matrimonios homosexuales y heterosexuales. Por otro lado, el censo nos aporta dos datos 
interesantes, que el número de parejas de hecho supera a las de derecho en el caso de parejas del mismo sexo y que dentro de estas últimas tres de cada cuatro no tienen hijos a su cargo.

\section{Conclusiones}

Nuestro propósito de partida era responder a la pregunta si, a diez años vista de su legalización, el matrimonio entre personas del mismo sexo era una realidad muy extendida por el territorio español y si se había ido consolidando en un periodo especialmente duro para la sociedad española. Los datos reflejan que su evolución se ajusta a la que se ha producido en otros países desarrollados: un primer año que bate récords de registros que luego aminoran manteniéndose, en unas frecuencias más o menos similares. De hecho, durante gran parte de los años de la crisis económica, se aprecia un ritmo de evolución un tanto similar al de las celebraciones de uniones entre personas de distinto sexo, así como una tendencia semejante a la que siguen otros parámetros demográficos como las tasas de fecundidad o de natalidad, el aumento de la edad media de maternidad, etc.

Por otra parte, a lo largo del presente análisis se ha puesto claramente de manifiesto la necesidad de incorporar un enfoque género cuando se analiza el impacto del matrimonio entre personas del mismo sexo en la sociedad española, tanto en lo que se refiere a sus tendencias, como a su reflejo territorial. En este sentido, cabe destacar que las uniones entre mujeres han ido ganando peso después de representar en los dos primeros años apenas uno de cada cuatro matrimonios celebrados. Dicho de otro modo, para el sexo femenino ha sido necesario un corto intervalo de tiempo para que las uniones entre mujeres se normalizasen como una práctica social en España, dado que es posible que estuvieran sujetas a una mayor contención en los primeros años de legalización.

El análisis geográfico del matrimonio entre personas del mismo sexo en España demuestra que su localización es bastante desigual. De manera general, ha tenido mayor protagonismo en gran parte de las provincias del litoral mediterráneo, en los dos archipiélagos y en la Comunidad de Madrid. Esta mayor difusión por el litoral, con mejores condiciones climáticas, es coincidente con lo sucedido en otros países como Estados Unidos o Francia y, en particular, en sus principales ciudades y enclaves turísticos. En gran medida, además, el matrimonio entre personas del mismo sexo es más numeroso en aquellas provincias más urbanizadas, con mayor presencia de población extranjera y bajos niveles de envejecimiento. La cartografía resultante guarda gran paralelismo con aquellos espacios donde la visibilidad homosexual es más amplia y normalizada, al menos en lo que a los varones se refiere. Ahora bien, no hay una coincidencia, al menos en esta escala espacial de análisis, con las zonas de mayor poder adquisitivo. En este sentido, cabría seguir indagando en la cuestión descendiendo en la escala de análisis a nivel intra-urbano, como se ha hecho en Estados Unidos, por ejemplo. Una vez más, cabe señalar un contraste entre el género de las uniones: la 
concentración es mayor en los matrimonios entre hombres que entre mujeres, como ocurre en otros países, y más localizada en las provincias del Mediterráneo.

Las fuentes utilizadas nos han proporcionado algunas pistas sobre los perfiles de los contrayentes de los matrimonios celebrados entre personas del mismo sexo. En gran medida se observa una gran correspondencia con personas de alto nivel de formación, de origen urbano y, desde el punto de vista etario, en una fase de primera madurez. En este sentido, en los dos sexos la edad a la que tiene lugar los enlaces es más tardía que en las uniones heterosexuales, ya de por sí elevada en comparación con la de años anteriores. Otro dato que llama especialmente la atención es la alta proporción de parejas de nacionalidad mixta en el matrimonio entre personas del mismo sexo, mucho mayor entre los hombres que entre las mujeres, lo que invita a una investigación de carácter cualitativo que podría esclarecer algunos otros aspectos de esta realidad demo-geográfica, al tiempo que contribuir, como el resto de estudios de este tipo, a consolidar una sociedad cada vez más diversa, tolerante e igualitaria.

Declaración responsable: Las/os autoras/es declaran que no existe ningún conflicto de interés en relación a la publicación de este artículo. El presente trabajo se expuso previamente en el XIX Coloquio Internacional de la AIDELF (Asociación Internacional de Demógrafos en Lengua Francesa), celebrado en junio de 2016 en la ciudad francesa de Estrasburgo. Alberto se encargó de la documentación y revisión bibliográfica; la redacción de los puntos 1, 3, 5 y las Conclusiones; y de la revisión y corrección de texto. José Antonio se encargó de la Búsqueda, tratamiento y explotación de datos estadísticos; la elaboración de gráficos y cartografía; la redacción de los puntos 2 y 4 ; y de la revisión y corrección final de texto. 


\section{Bibliografía}

Andersson, G., Noack, T., Seierstad, A., \& Weedon-Fekjaer, H. (2006). The demographics of same-sex marriages in Norway and Sweden. Demography, 43(1), 79-98.

Banens, M. (2016). Étudier le couple de même sexe avec le recensement de la population. Paper presented at the XIX Colloque International de I'AIDELF: Configurations et dynamiques familiales, AIDELF e Institut de Démographie de I'Université de Strasbourg. Retrieved from https://colloque.aidelf.org/documents/1692.pdf

Banens, M. (2010). Mariage et partenariat de même sexe en Europe. Vingt ans d'expérience. Politiques Sociales et Familiales, 99, 73-84.

Belliot, N. (2013). L'impact démographique du pacte civil de solidarité et le mariage pour tous. Bulletin du CERFAP, 15, 3-4.

Belliot, N. (2009). Démographie des mariages et unions entre personnes de même sexe. L'accès au mariage favorise t'il la légalisation des unions homosexuelles? Paper presented at the XXVI IUSSP International Population Conference, The International Union for the Scientific Study of Population. Retrieved from http://iussp2009.princeton.edu/papers/92615

Black, D., Gates, G., \& Lowell, T. (2002). Why do gay men live in San Francisco? Journal of Urban Economics, 51, 54-76.

Black, D., Gates, G., Sanders, S., \& Lowell, T. (2000). Demographics of the Gay and Lesbian Population in the United States: Evidence from Available Systematic Date Sources. Demography, 39(2), 139-154.

Castro Martín, T. (2015). Nuevas familias para un nuevo siglo. In C. Torres Albero (Ed.), España 2015. Situación social (pp. 303-314). Madrid: Centro de Investigaciones Sociológicas.

Castro Martín, T., Martín García, T., Abellán, A., Pujol, R., \& Puga, D. (2015). Tras las huellas de la crisis económica en la demografía española. Panorama Social, 22, 43-60.

Castro Martín, T., \& Seiz Puyuelo, M. (2014). La transformación de las familias en España desde una perspectiva socio-demográfica (VII Informe sobre exclusión y desarrollo social en España). Madrid: Fundación Foessa.

Cortina, C. (2016). Demografía de las parejas homosexuales en España. Revista Española de Investigaciones Sociológicas, 153, 3-22.

Cortina, C., \& Cabré, A. (2010). Las uniones homosexuales en España. Una caracterización sociodemográfica a partir del Censo 2001. Papers-Revista de Sociología, 95(3), 565-583. 
Domínguez Folgueras, M. (2011). Diez cambios en las parejas españolas: 1995-2006. Madrid: Centro de Investigaciones Sociológicas.

Fernández Salinas, V. (2008). ¿Un planeta fuera del armario? La visibilidad gay como objeto de estudio geográfico. Scripta Nova, XII(270). Retrieved from http://www.ub.edu/geocrit/sn/sn270/sn-270-43.htm

Fernández Salinas, V. (2007). Visibilidad y escena gay masculina en la ciudad española. Documents d'Análisi Geogràfica, 49, 139-160.

Fernández Salinas, V. (2007). Comunidad gay y espacio en España. Boletín de la Asociación de Geógrafos Españoles, 43, 241-260.

Festy, P. (2006). La légalisation des couples homosexuels en Europe. Population, 51, 493-531.

García Escalona, E. (2000). Del armario al barrio: aproximación a un nuevo espacio urbano. Anales de Geografía de la Universidad Complutense, 20, 437-449.

Gimeno, B., \& Barrientos, V. (2009). La institución matrimonial después del matrimonio homosexual. Íconos, 35, 19-30.

Gimeno, B. (2003). El amor que no osa decir su nombre. La invisibilidad de las lesbianas. A distancia, 21(3), 131-136.

Giraud, C. (2013). Le Village gai de Montréal. Une aventure urbaine minoritaire. Espaces et Sociétés, $154,33-48$.

Goicoechea Gaona, M. A., Fernández Guerrero, O., Clavo Sebastián, M. J., \& Álvarez Terán, R. (2015). Chicas que entienden. In-visibilidad lesbiana. Barcelona-Madrid: Egales.

Grau Rebollo, J. (2016). Nuevas formas de familia. Ámbitos emergentes. Barcelona: Edicions Bellaterra.

Jurado, T. (2008). Las nuevas familias españolas. In J. J. González, \& M. Requena, M. (Eds.), Tres décadas de cambio social en España (pp. 59-88). Madrid: Alianza Editorial.

Hughes, H. (2003). Marketing gay tourism in Manchester: New market for urban tourism or destruction of 'gay space'? Journal of Vacation Marketing, 9(2), 152-163.

Jambu, M., \& Lebeaux, M. O. (1983). Cluster analysis and data analysis. Amsterdam: Elsevier Science Ltd.

Kanai, J. M. (2014). Whither queer world cities? Homo-entrepreneurialism and beyond. Geoforum, $56,1-5$. 
Léobon, A. (2006). Champs de libertés et constructions de territoires et bisexuels en France et au Québec. In R. Séchet, \& V. Veschambre (Dirs.), Penser et faire la géographie sociale (pp. 277294). Rennes: Presses Universitaires de Rennes.

Leroy, S. (2006). Le Paris gay. Éléments pour une géographie de I'homosexualité. Annales de Géographie, 646, 579-601.

Lucas Marín, A. (2010). La institución familiar. In A. Lucas Marín (Ed.), La realidad social: transformaciones recientes en España (pp. 142-179). Pamplona: Ediciones de la Universidad de Navarra.

Mazuy, M., Barbieri, M., Breton, D., \& D’albis, H. (2015): L'évolution démographique récente de la France et ses tendances depuis 70 ans. Population, 70(3), 417-486.

Melián González, A., Moreno Gio, S., \& Araña, J. (2011). Gay tourism in a sun and beach destination. Tourism Management, 32(5), 1027-1037.

Paradero Huerta, I. (2013). Nupcialidad de parejas del mismo sexo: igualdad legal sin igualdad social. Paper presented at the XI Congreso Español de Sociología, Universidad Complutense de Madrid. Retrieved from http://www.fes-sociologia.com/nupcial1405ad-de-parejas-del-mismo-sexoigualdad-legal-sin-igualdad-social/congress-papers/1405/

Pichardo Galán, J. I. (2009). (Homo)sexualidad y familia: cambios y continuidades al inicio del tercer milenio. Política y Sociedad, 46, 143-160.

Platero, R. (2007). Entre la invisibilidad y la igualdad formal: perspectivas feministas ante la representación del lesbianismo en el matrimonio homosexual. In F. Rodríguez, \& A. Simonis (Eds.), Cultura, Homosexualidad y Homofobia (pp. 85-106). Madrid: Laertes.

Remiggi, F. (1998). Le village gai de Montréal: entre le ghetto et l'espace identitaire. In I. Demczuk, \& F. Remiggi (Dirs.), Sortir de l'ombre (pp. 267-289). Montreal: VLB Éditeur.

Requena y Díaz de Revenga, M. (2010). Los cambios familiares en España y sus implicaciones. Revista del Ministerio de Trabajo e Inmigración, $n^{\circ}$ extraordinario 1, 47-68. Retrieved from http://www.empleo.gob.es/es/publica/pub_electronicas/destacadas/revista/anyo2010/R evista_Extra_Seguridad_Social.pdf

Requena, M. (2008). Bases demográficas de la sociedad española. In J. J. González, \& M. Requena, M. (Eds.), Tres décadas de cambio social en España (pp. 29-58). Madrid: Alianza Editorial.

Santos Solla, X. M. (2002). Espacios disidentes en los procesos de ordenación territorial. Documents d'Análisi Geogràfica, 40, 69-104. 
Soriano Martínez, E. (2011). El matrimonio homosexual en Europa. Revista Boliviana de Derecho, $12,204-216$. 